\title{
MÉTODO PARA A ESTIMATIVA DA COBERTURA DO SOLO E DA ALTURA DO DOSSEL DE ALGUMAS CULTURAS DE VERÃO(1)
}

\author{
G. ROLOFF(2) \& O.J.BERTOL ${ }^{(3)}$
}

\begin{abstract}
RESUMO
O uso de modelos de erosão requer dados sobre a cobertura do solo pelo dossel das culturas e sua altura. Equações que descrevem as relações entre a biomassa aérea e a fração do solo coberta pelo dossel e a altura mediana das culturas do algodão, batata, feijão, milho e soja foram desenvolvidas a partir de dados, coletados em áreas comerciais de diversos municípios do Estado do Paraná, durante a safra 94/95. Quinzenal mente, foram coletadas a fração coberta pelo dossel, sua altura e a biomassa aérea. 0 rendimento das culturas foi determinado por amostragem manual. As relações uti lizadas, que usam variáveis proporcionais, resultaram em coeficientes de determinação $\left(R^{2}\right)$ de 0,87 , ou maiores, e demandam dados si mples e, geralmente, disponíveis. A biomassa aérea durante a safra foi estimada com precisão similar, tanto por unidades de calor acumuladas quanto por dias após plantio. A forma da relação entre biomassa aérea ecobertura dependeu da cultura, sendo a forma exponencial mais adequada à cultura do feijão e a forma logística às do mi lho e soja. Ambas as formas foram apropriadas para as culturas do algodão e da batata. A altura do dossel foi estimada a partir da biomassa aérea também com boa precisão $\left(R^{2} \geq 0,87\right)$.
\end{abstract}

Termos de indexação: cobertura do solo pelo dossel, altura das culturas, biomassa aérea, modelagem da erosão.

\section{SUMMARY: METHOD TO ESTIMATE CANOPY COVER AND HEIGHT OF SEVERAL SUMMER CROPS}

Utilization of soil erosion models requires data on soil canopy cover and crop height. Equations to describe the relationship of aboveground biomass to soil canopy cover and height of the corn, cotton, dry bean, potato and soybean crops were developed from data

(1) Trabal ho financiado pela FAO (projeto GCP/RLA/107/J PN), realizado em cooperação entre a UFPR, a EMATER-PR ea FUNPAR. Recebido para publicacão em novembro de 1996 e aprovado em dezembro de 1997.

(2) Professor Adjunto, Departamento de Solos, Universidade Federal do Paraná. Caixa Postal 2959, CEP $80001-970$ Curitiba (PR). Bolsista CNPq.

(3) Engenheiro-Agrônomo, Coordenador Estadual de Recursos Naturais, EMATER (PR). Rua da Bandeira, 570, CEP 80035-270 Curitiba (PR). 
collected at farm plots in several municipalities of the State of Paraná during the 94/ 95 crop year. Canopy cover and height, and aboveground bi omass werecollected at fifteen-day intervals. Yield was determined by manual harvest. The relations utilized, which employ proportional variables, resulted in determination coefficients $\left(R^{2}\right)$ of 0.87 or higher, and requi resimpleand generally available data. Biomass accumulati on during the season was estimated with similar precision by either the cumulative heat units or days after seeding. The form of the relation between aboveground biomass and canopy cover depended on the crop. The exponential form was moreappropriatefor thedry bean crop and thelogistic form for thecorn and soybean crops. Either form semed adequatefor the cotton and potato crops. Canopy height was also estimated from the aboveground biomass with good precision $\left(R^{2} \geq 0,87\right)$.

Index terms: canopy cover, canopy height, aboveground biomass, erosi on modelling.

\section{NTRODUÇÃO}

A cobertura do sol o pel o dossel das plantas reduz ou elimina o efeito do impacto das gotas de chuva sobre a superfície do solo, minimizando, assim, a formação do sel o superficial ea desagregação do sol o. I sso torna a cobertura eficiente na proteção do solo por reduzir, direta ou indiretamente, as perdas de solo por erosão entre sulcos, em sulcos e em canais. A quantificação da variação dessa cobertura durante a safra, em solos sob intensa utilização agrícola, é essencial na avaliação do grau de proteção contra a erosão gerado pelas diversas culturas. A eficiência dessa proteção está relacionada com a altura do dossel (Wischmeier \& Smith, 1978), sendo sua quantificação similarmente necessária.

A quantificação da cobertura e da altura do dossel é requerida pelos modelos matemáticos de estimativa da erosão. O estabel ecimento de um banco de dados sobre a proteção pelo dossel das culturas viabilizou a utilização rotineira do model oSLEMSA (Soil Loss Estimator for Meridional and Southern Africa) para a avaliação da erosão em Zimbabwe (Elwell \& Stocking, 1982). O model oRUSLE (Revised Universal Soil Loss Equation; Renard et al., 1991) utiliza um banco de dados interno com a proporção da superfíce coberta e a altura mediana do dossel das culturas. Os model os EPIC (E rosion Productivy Impact Calculator; Williams et al., 1989) e WEPP (Water Erosion Prediction Project; Alberts et al., 1989) geram matematicamente tais parâmetros.

Diversos fatores naturais eantrópicos controlam o grau de cobertura do sol o pel o dossel das culturas. Para que tais modelos matemáticos possam ser usados rotineiramente, contudo, é necessário que a proteção por esta cobertura possa ser estimada de maneira simplificada, usando dados disponíveis ou de fácil obtenção, considerando os efeitos do ambiente sobre o desenvolvimento das culturas. Além disso, a possibilidade de estimar a variação da cobertura e seu efeito sobre a er osão pode auxiliar o estabel ecimento de planos de manejo, cujos objetivos incluam a conservação do sol o. O presente trabal ho teve como objetivo desenvol ver rel ações matemáticas que permitam a estimativa da fração coberta e da altura mediana do dossel das culturas do algodão, batata, feijão, milho esoja, utilizando dados simples e amplamente disponíveis. Tais relações facilitarão o uso futuro de model os matemáticos de erosão e o estabelecimento de estratégias de manejo para a minimização da erosão do solo.

\section{Base teórica}

A cobertura do solo pelo dossel depende, principalmente, da espécie, da biomassa aérea e da densidade populacional. Desses fatores, a biomassa esua variação no tempo dificilmente são conhecidas em situações práticas de campo. Para contornar isto, o primeiro passo é estimar a biomassa aérea total máxima da cultura (B $\mathbf{m x}$ ) através de sua relação com o rendimento do produto comercial $(\mathbf{P})$, usando oíndice de colheita (Ic) (Sharpley \& Williams, 1990), conforme a expressão

$$
B m x=\frac{P}{I c}
$$

Para generalizar e simplificar as relações, é necessário supor que: (1) o rendimento da cultura seja resultado da integração dos fatores controladores do seu crescimento, e (2) eventuais déficits dos fatores ambientais sejam distribuídos, igualmente, durante a safra. Com isso, a biomassa aérea durantea safra $\left(\mathbf{B m}_{\mathbf{d}}\right)$, em qual quer dia entre o plantio ea maturidadefisiológica, podeser descrita como uma proporção de $\mathbf{B} \mathbf{m x}$, na forma de

$$
B p_{d}=\frac{B m_{d}}{B m x}
$$

em que $\mathbf{B} \mathbf{p}_{\mathbf{d}}$ é a biomassa aérea proporcional no dia d. 
A estimativa de $\mathbf{B} \mathbf{p}_{\mathbf{d}}$ deve levar em consideração as variações climáticas e a data de plantio, através de uma variável climática $\left(\mathbf{V}_{\mathbf{c}}\right)$. Tal estimativa pode ser feita através de uma função de crescimento logística, adaptada de Kiniry et al. (1992), descrita por

$$
B p_{d}=\frac{V c_{d}}{V c_{d}+e^{\left(\alpha-\beta V c_{d}\right)}}
$$

Alternativamente, pode ser utilizada uma função de crescimento exponencial (Alberts et al., 1989), descrita por

$$
B p_{d}=V c_{d} \chi
$$

Os parâmetros $\alpha, \beta$ e $\chi$ são específicos à cultura e são obtidos pelo ajuste de regressão não-linear (e.g. Seber \& Wild, 1989) a partir de dados experimentais.

Similarmente à biomassa, o uso de uma $\mathbf{V} \mathbf{c}_{\mathbf{d}}$ proporcional generaliza e simplifica as relações. $\mathrm{Na}$ ausência de déficit hídrico, a velocidade de crescimento das culturas é controlada, principalmente, pelo calor. Assim, para considerar diferenças em variedades e climas regionais, $\mathbf{V c}_{\mathbf{d}}$ pode ser avaliada pelo conceito de calor acumulado proporcional $\left(\mathbf{C p}_{\mathbf{d}}\right)$ (Alberts et al., 1989), na forma de

$$
V c_{d}=C p_{d}=\frac{C a_{d}}{C x}
$$

em que $\mathbf{C x}$ é o número de unidades de calor acumulado necessário para a maturação da cultura. O número de unidades de cal or acumuladas $\left(\mathbf{C a}_{\mathbf{d}}\right)$, para qualquer dia entre o plantio e a maturação, é calculado por

$$
C a_{d}=\sum_{1}^{d}\left[\left(\frac{T x_{d}+T n_{d}}{2}\right)-T b\right]
$$

em que d édias após o plantio ou após a emergência, $\mathbf{T} \mathbf{x}_{\mathbf{d}}$ e $\mathbf{T n}_{\mathbf{d}}$ são as temperaturas máxima e mínima diárias, e Tb é a temperatura-base para a cultura, abaixo da qual não há crescimento.

Existe a possibilidade de os usuários potenciais não terem acesso a valores diários de $\mathbf{T} \mathbf{x}_{\mathbf{d}}$ e $\mathbf{T} \mathbf{n}_{\mathbf{d}}$, ou de Cx. Para contornar essa dificuldade, a equação [5] pode ser substituída por

$$
V c_{d}=D p l_{d}=\frac{D l_{d}}{D l x}
$$

ou

$$
V c_{d}=D p e_{d}=\frac{D e_{d}}{D e x}
$$

nas quais o dia proporcional ( $\mathbf{D p} \mathbf{l}_{\mathbf{d}}$ ou $\mathbf{D p e}_{\mathbf{d}}$ ) é calculado apenas pelo número de dias após plantio $\left(\mathbf{D I}_{\mathbf{d}}\right)$ ou após emergência $\left(\mathbf{D e}_{\mathbf{d}}\right)$, em relação ao número de dias necessários para atingir a maturidade fisiológica (DIx ou Dex). Os valores de DIx ou Dex são, normalmente, conhecidos para as variedades comumente plantadas. O uso de dias após emergência pode melhorar o ajuste das relações porque podem haver diferenças da data deemergência entre anos ou locais, dependendo da água do solo usualmente disponível para o processo de germinação. Similarmente, o cal or acumulado pode ser calculado a partir do plantio $\left(\mathbf{C} \mathbf{p l}_{\mathbf{d}}\right)$ ou da emergência $\left(\mathbf{C p e}_{\mathbf{d}}\right)$ da cultura.

A partir da estimativa de $\mathbf{B m}_{\mathbf{d}}$, a fração do solo coberta pelo dossel no dia $\mathbf{d}\left(\mathbf{F C}_{\mathbf{d}}\right)$ é calculada pela expressão

$$
F_{\mathbf{C}_{\mathbf{d}}}=\left(\mathbf{1}-\mathbf{e}^{-\varepsilon B m} \mathbf{d}\right) \delta
$$

(modificada de Alberts et al., 1989) em que $\delta$ e $\varepsilon$ são parâmetros específicos à cultura, também obtidos por meio do ajuste de regressão não-linear a partir de dados experimentais. Tal relação é, geralmente, válida no intervalo entre plantio efinal da floração. $\mathrm{Na}$ maioria das culturas, a cobertura pelo dossel tende a decrescer após esse estágio em virtude da progressiva senescência das folhas, apesar de a biomassa aérea aumentar até a maturidade fisiológica.

O efeito da cobertura viva sobre a erosão depende, também, da altura do dossel das culturas, representada pela altura mediana de queda das gotas formadas no dossel $\left(\mathbf{A}_{\mathbf{d}}\right)$. Essa altura é relacionada com a biomassa aérea por

$$
A_{d}=\left(1-e^{-\phi B m_{d}}\right) A x
$$

(Alberts et al., 1989) em que Ax éa altura mediana máxima do dossel e $\phi$ é um parâmetro específico à cultura, obtida também pel o ajuste de regressão nãolinear a partir de dados experimentais.

\section{MATERIAL E MÉTODOS}

Os dados experimentais das culturas foram coletados por extensionistas da EMATER (PR), durante a safra 94/95, em áreas comerciais de diversos locais do Estado do Paraná (Quadro 1), representativos da diversidade edafodimática, do manejo e da produtividade dessa região brasileira. As propriedades sel eci onadas estão numa distância de até $20 \mathrm{~km}$ de uma estação meteorológica.

Em cada propriedade, foi escolhida uma gleba visual mente homogênea em torno de 0,4 ha, na qual foram coletadas a biomassa aérea, a fração da superfície do solo coberta pela cultura e a altura 
Quadro 1. Localização e características agronômicas das culturas monitoradas

\begin{tabular}{|c|c|c|c|c|c|c|}
\hline \multirow[b]{2}{*}{ Cultura } & \multirow[b]{2}{*}{ Município } & \multirow[b]{2}{*}{ Localização } & \multirow[b]{2}{*}{ Glebas } & \multirow[b]{2}{*}{ Variedades } & \multicolumn{2}{|c|}{ E spaçamento $^{(1)}$} \\
\hline & & & & & Entre linhas & Entre plantas \\
\hline & \multicolumn{4}{|c|}{ un. } & \multicolumn{2}{|c|}{$\mathrm{m}$} \\
\hline Algodão & Pitangueiras & 231ㅇ'S 5130'W & 2 & IAC-20 & $0,8-0,9$ & $0,13-0,14$ \\
\hline \multirow[t]{3}{*}{ Batata } & Araucária & 253'S 4923'W & 3 & Contenda e Araucária & 0,7 & $0,25-0,33$ \\
\hline & Guarapuava & 25인 5 512ㅇ'W & 2 & Bintje e Monalisa & 0,8 & $0,09-0,13$ \\
\hline & Araucária & $25 \div 37^{\prime} \mathrm{S} 49 \div 23^{\prime} \mathrm{W}$ & $3^{(2)}$ & IAPAR-44 & 0,6 & $0,08-0,14$ \\
\hline \multirow[t]{3}{*}{ F eijão } & Guapirama & 230'S 495'W & 3 & IAPAR-14 & 0,5 & $0,07-0,09$ \\
\hline & Pato Branco & 26013'S 5239'W & $1(2)^{(3)}$ & IAPAR-14 & $0,4-0,5$ & $0,07-0,08$ \\
\hline & Cascavel & 245ㅇ'S 532ㅇ'W & 3 & Pioneer 3072 & $0,8-0,9$ & $0,17-0,20$ \\
\hline \multirow[t]{2}{*}{ Milho } & Guapirama & 23907'S 4958'W & 3 & BR-201, AG-510 e Germinal-600 & 0,9 & 0,20 \\
\hline & Pato Branco & 2613'S 5239'W & $(2)$ & Germinal-85 e FT-9043 & 0,9 & $0,17-0,20$ \\
\hline \multirow[t]{2}{*}{ Soja } & Ponta Grossa & 25007'S 5010'W & 1 & FT-10 & 0,4 & 0,06 \\
\hline & Rolândia & 231ㅇ'S 5120'W & 2 & FT-Abyara e FT-5 & $0,4-0,5$ & $0,06-0,08$ \\
\hline
\end{tabular}

(1) Amplitude dos val ores encontrados. ${ }^{(2)}$ Inclui uma gleba com plantio “da seca” (fevereiro-maio). ${ }^{\text {(3) }}$ Parênteses indicam o número de glebas utilizadas como testes independentes, não incluídas na geração das constantes.

máxima das plantas, com três repetições, em intervalos, aproximadamente, quinzenais. Nessa gleba, também foi determinado o rendimento (produto comercial ), em uma subárea de 10 m de comprimento por uma linha da cultura, com três repetições.

A cobertura foi medida por um aparato descrito em Stocking (1985), com algumas modificações. Esse aparato consisteem uma estrutura com uma travessa horizontal, com dez orifícios para visualização, a cerca de 1,5 m de altura do solo, e dois suportes verticais. Esses orifícios, espaçados em $0,1 \mathrm{~m}$, são dotados de dois pares de fios de alinhamento que permitem a materialização de um ponto durante a visualização pelo operador, de cima para baixo. Para os casos em que havia partes da cultura acima da travessa horizontal do aparato, era colocado um espel ho abaixo da travessa para permitir a visualização de baixo para cima. Os suportes da estrutura eram encaixados sobre uma moldura-suporte, em forma de paralelogramo com ângulos variáveis elados de $1 \mathrm{~m}$, com filas dedez furos espaçados de 0,1 $\mathrm{m}$ em dois lados opostos. Os ângulos do paralelograma eram ajustados, para que os lados com furos ficassem centralizados em entrelinhas da cultura. A estrutura com os orifícios era então encaixada nos furos do suporte erealizadas as leituras. Os dez orifícios da travessa horizontal eram visualizados na posição de cada um dos dez furos da moldura, totalizando, assim, 100 pontos de leitura, dispostos em um espaçamento de 0,1 por $0,1 \mathrm{~m}$, ou menor, dependendo das dimensões do paral el ogramo. Um ponto era marcado como "coberto", quando qualquer parte do dossel da cultura seencontrava sob ou sobreeste. A pós a leitura, a al tura total das plantas era medida, ea biomassa aérea col etada, excetuandose as partes das plantas que ultrapassavam o perímetro da moldura. A biomassa coletada era transferida para estufa a $60^{\circ} \mathrm{C}$ dentro de $48 \mathrm{~h}$ para determi nação da massa seca, exceto em al guns casos, quando foi seca ao ar e, posteriormente, em estufa.

Os valores médios por propriedade, de cada data de amostragem, foram usados na determinação das variáveis das diversas equações. A maturação foi definida como o dia em que a biomassa aérea atingiu seu maior valor (Bmx), determinada em uma curva polinomial deterceiro grau, gerada a partir dos dados de DI (x) e Bm (y). Os outros valores máximos (Cx, DIx, Dex e Ax) foram então calculados em relação a este dia, usando dados de $\mathbf{T} \mathbf{x}$ e $\mathbf{T n}$ da estação meteorológica próxima, quando necessário. A Tb utilizada para as culturas foi de $1^{\circ}{ }^{\circ} \mathrm{C}$, à exceção da batata, cujo valor foi de 7C (Dumesnil, 1994). A altura mediana foi considerada como sendo a metade da altura total medida em cada ocasião. As constantes das equações foram determinadas por regressão nãolinear, através da técnica dos mínimos quadrados, usando o método iterativo quasi-Newtoniano, pelo programa estatístico SYSTAT (SYSTAT, Inc., 1993).

Comoteste das equações geradas para as culturas do feijão e do milho, a fração coberta calculada foi comparada com a medi da em glebas, cujos dados não foram usados na determinação das constantes (Quadro 1). Nesse processo, os dados de $\mathbf{P}, \mathbf{D I x}, \mathbf{C x}, \mathbf{T x}$ e Tn foram col etados do campo ou da estação meteorológica. Tais dados permitiram o cálcul o, em seqüência, de $\mathbf{V c}_{\mathbf{d}}$ e $\mathbf{B p}_{\mathbf{d}}$, com as constantes específicas às culturas testadas (Quadros 3 e 4) e com os val ores médios de Ic (Quadro2). O val or de Bm foi cal culado pela equação [4] para o feijão e equação [3] para o milho. Para as demais culturas, não havia gl ebas adicionais para teste independente. 


\section{RESULTADOS E DISCUSSÃO}

Os valores de Ic obtidos (Quadro 2) estão geralmente próximos dos reportados na literatura, à exceção do obtido para a batata no município de Guarapuava, no qual o Ic foi cerca de $25 \%$ inferior ao da literatura. A razão para esse baixo valor de Ic nãofoi determinada, mas pode estar relacionada com déficits hídricos durante o desenvolvimento da cultura. Na maior parte dos casos, Ic apresentou alta variabilidade, devida, provavelmente, à utilização pelos agricultores de variedades diversas e, em al guns casos, de sementes próprias de feijão ebatata. Tais resultados indicam que os valores de Ic, ideal mente, devem ser conhecidos para a variedade. Os valores da literatura, contudo, são apropriados para utilização prelimi nar, uma vez que são próximos dos medidos.

O uso de parâmetros proporcionais para quantificar a relação entre o tempo, expresso por $\mathbf{C p l} \mathbf{l}_{\mathbf{d}}, \mathbf{C p e}_{\mathbf{d}}, \mathbf{D p l} \mathbf{d}_{\mathbf{d}}$ ou $\mathbf{D p e}_{\mathbf{d}}$, e a acumulação da biomassa aérea, resultou em relações estreitas (Quadro 3), conforme os altos coeficientes de determinação ( $\mathbf{R}^{\mathbf{2}}$ ) obtidos. Para a batata, não foram calculados parâmetros relacionados com dias após plantio, porque, em um dos municípios (Araucária), as plantas levaram cerca de um mês para emergirem por causa da falta de chuvas. Como isto, normalmente, nãotraz conseqüência ao rendimento dessa cultura, optou-se por utilizar somente dias após emergência. As diversas formas da relação biomassa $X$ tempo são independentes do local de amostragem, exceto para a cultura da batata, indicando sua generalidade e independência dos
Quadro 2. Índices de colheita (Ic) para cinco culturas de verão no Estado do Paraná

\begin{tabular}{|c|c|c|c|c|c|}
\hline Cultura & Município & $n^{(1)}$ & $I \mathbf{C}^{(2)}$ & $\begin{array}{l}\mathbf{C V}^{(2)} \\
(\%)\end{array}$ & $\begin{array}{c}\text { Ic } \\
\text { literatura }\end{array}$ \\
\hline \multirow[t]{2}{*}{ Algodão } & Rolândia & 2 & 0,38 & - & 0,40 \\
\hline & Araucária & 3 & 0,94 & 39 & \\
\hline \multirow[t]{3}{*}{ Batata } & Guarapuava & 2 & 0,72 & - & $0,95-1,12$ \\
\hline & Ambos & 5 & 0,91 & 33 & \\
\hline & Araucária & 3 & 0,28 & 23 & \\
\hline \multirow[t]{3}{*}{ Feijão } & Guapirama & 3 & 0,31 & 49 & Não-disponível \\
\hline & Ambos & 6 & 0,30 & 36 & \\
\hline & Cascavel & 3 & 0,49 & 8 & \\
\hline \multirow[t]{2}{*}{ Milho } & Guapirama & 3 & 0,52 & 18 & 0,50 \\
\hline & Ambos & 6 & 0,51 & 13 & \\
\hline Soja & $\begin{array}{l}\text { Ponta Grossa } \epsilon \\
\text { Rolândia }\end{array}$ & 3 & 0,32 & 6 & 0,30 \\
\hline
\end{tabular}

outros fatores controladores do crescimento. Apesar de não se ter verificado diferença estatística entre os valores por município, os parâmetros e o $\mathbf{R}^{\mathbf{2}}$ obtidos para a batata são relativamente distintos entre os dois municípios amostrados, provavel mente em razão dos diferentes tipos das variedades plantadas. As variedades usadas no município de

Quadro 3. Parâmetros $\left(\alpha\right.$ e $\beta$ ) e coeficientes de determinação ajustado $\left(R^{2}\right)$ das equações, relacionando tempo e biomassa aérea, para cinco culturas de verão no Estado do Paraná

\begin{tabular}{|c|c|c|c|c|c|c|c|c|c|c|c|c|c|c|c|c|c|c|c|c|c|}
\hline \multirow{3}{*}{ Cultura } & \multirow{3}{*}{$n^{(3)}$} & \multicolumn{5}{|c|}{$V c_{d}=C_{p l}{ }_{d}^{(1)}$} & \multicolumn{5}{|c|}{$V_{c_{d}}=C_{p e}{ }_{d}^{(1)}$} & \multicolumn{5}{|c|}{$V c_{d}=D p l_{d}{ }^{(2)}$} & \multicolumn{5}{|c|}{$V c_{d}=D p e_{d}^{(2)}$} \\
\hline & & \multicolumn{3}{|c|}{ Equação [3] } & \multicolumn{2}{|c|}{ Eq. [4] } & \multicolumn{3}{|c|}{ Equação [3] } & \multicolumn{2}{|c|}{ Eq. [4] } & \multicolumn{3}{|c|}{ Equação [3] } & \multicolumn{2}{|c|}{ Eq. [4] } & \multicolumn{3}{|c|}{ Equação [3] } & \multicolumn{2}{|c|}{ Eq. [4] } \\
\hline & & $\alpha$ & $\beta$ & $\overline{\mathbf{R}^{2}}$ & $\chi$ & $\mathbf{R}^{2}$ & $\alpha$ & $\beta$ & $\overline{\mathbf{R}^{2}}$ & $\chi$ & $\mathbf{R}^{2}$ & $\alpha$ & $\beta$ & $\mathbf{R}^{2}$ & $\chi$ & $\mathbf{R}^{2}$ & $\alpha$ & $\beta$ & $\mathbf{R}^{2}$ & $\chi$ & $\mathbf{R}^{2}$ \\
\hline न & 20 & 1,76 & 3,49 & 0,93 & 1,68 & 0,92 & 1,34 & 3,03 & 0,93 & 1,52 & 0,92 & 1,76 & 3,56 & 0,92 & 1,64 & 0,92 & 1,33 & 3,09 & 0,93 & 1,48 & 0,92 \\
\hline $\begin{array}{l}\text { Batata: } \\
\text { Araucária }\end{array}$ & 13 & $-^{(4)}$ & - & - & - & - & 1,84 & 4,32 & 0,77 & 1,29 & 0,78 & - & - & - & - & - & 1,92 & 4,51 & 0,79 & 1,28 & 0,80 \\
\hline Guarapuava & 9 & - & - & - & - & - & 6,61 & 9,94 & 0,96 & 2,29 & 0,97 & - & - & - & - & - & 5,20 & 8,66 & 0,97 & 1,94 & 0,94 \\
\hline Geral $^{(5)}$ & 22 & - & - & - & - & - & 2,47 & 4,83 & 0,81 & 1,58 & 0,83 & - & - & - & - & - & 2,50 & 5,09 & 0,84 & 1,48 & 0,85 \\
\hline Feijão & 31 & 5,36 & 7,57 & 0,94 & 2,78 & 0,97 & 4,35 & 6,69 & 0,94 & 2,24 & 0,97 & 5,61 & 8,02 & 0,94 & 2,61 & 0,96 & 4,29 & 6,82 & 0,95 & 2,07 & 0,96 \\
\hline Milho & 40 & 4,81 & 8,14 & 0,97 & 1,89 & 0,93 & 3,90 & 7,17 & 0,97 & 1,67 & 0,93 & 4,69 & 7,86 & 0,97 & 1,93 & 0,97 & 3,80 & 6,94 & 0,97 & 1,69 & 0,94 \\
\hline Soja & 22 & 4,51 & 7,21 & 0,87 & 2,10 & 0,86 & 3,67 & 6,39 & 0,88 & 1,83 & 0,87 & 4,45 & 7,40 & 0,90 & 1,96 & 0,87 & 3,61 & 6,56 & 0,90 & 1,71 & 0,88 \\
\hline
\end{tabular}

${ }^{(1)} \mathbf{C p l}_{d}$ é o calor acumulado cal culado entre a data do plantio e da maturação e $\mathbf{C p e}_{\mathbf{d}}$ é entre a data de emergência e da maturação. ${ }^{(2)} \mathbf{D p} / \mathbf{d}$ é o dia proporcional após o plantio e Dped após a emergência. ${ }^{(3)} \mathbf{n}$ é o número de pontos usados na regressão. ${ }^{(4)}$ não-determinado. ${ }^{(5)}$ Geral para os dois municípios. 
Araucária visam à venda in natura ao consumidor, enquanto as do município de Guarapuava visam à sua utilização industrial .

Em relação à forma da equação relacionando tempo e biomassa aérea, observam-se diferenças muito pequenas entre os val ores de $\mathbf{R}^{\mathbf{2}}$ das equações [3] e [4] para as culturas do al godão, batata e soja. Para as culturas do feijão e do milho, no entanto, as diferenças são maiores. A aval iação gráfica do ajuste (Figura 1) indica que os maiores valores de $\mathbf{R}^{\mathbf{2}}$ estão associados a um melhor ajuste ao longo de toda a curva, com a equação [4] (exponencial) sendo superior para a cultura do feijão, enquanto a equação [3] (logística) é superior para a cultura do milho. No caso da cultura da soja, apesar de valores similares de $\mathbf{R}^{\mathbf{2}}$, a equação [3] mostra mel hor ajuste no início da cultura (Figura 1). Nessa fase, é importante que a estimativa de cobertura seja relativamente mais precisa porque é uma fase com alto potencial de perdas por erosão devidas à pequena porém crescente proteção oferecida pelo dossel no início do seu desenvolvimento. Portanto, também para a cultura da soja, a equação [3] é mais apropriada. Análise gráfica similar para as culturas do al godão e da batata indicou que o ajuste na fase inicial é similar entreas duas equações, razão por que podem ser usadas indistintamente.

O uso de cal or acumulado ( $\mathbf{C} \mathbf{p l}_{\mathbf{d}}$ ou $\mathbf{C} \mathbf{p e}_{\mathbf{d}}$ ) ou dias após plantio (Dpl ou $\mathbf{D p e}$ ) resultou em valores de $\mathbf{R}^{2}$ muito próximos, para todas as culturas. Parcialmente, tal resultado deve-se ao método que gerou ambos os valores, com os cál cul os sendo feitos em relação ao dia em que Bx foi atingido, e ao uso de valores proporcionais. A contagem do tempo, a partir da data do plantio ou da emergência, não resultou em diferenças marcantes entre os valores de $\mathbf{R}^{2}$ (Quadro 3). Praticamente, torna-se desnecessário o conhecimento da data de emergência para o uso dessas relações, no âmbito deste estudo, exceto para a cultura da batata.
A relação da biomassa aérea com a fração do sol o coberta pel o dossel foi apropriadamentedescrita pela equação [9], julgando-se pelos valores de $\mathbf{R}^{2}$ relativamente altos (Quadro 4). Na literatura, essa relação é, normalmente, expressa com a constante $\delta$ ausente, o que a iguala a 1 (e.g. Alberts et al., 1989). Os resultados deste trabalho demonstram, porém, que $\delta$ se aproximou do valor unitário apenas para as culturas do feijão e da soja, e mais distante, da batata. Valores de $\delta$ menores que a unidade fazem com que a curva apresente um patamar distinto, no qual incrementos de biomassa aérea não implicam aumentos na fração coberta, como exemplificado para o mil ho na figura 2. O valor unitário, por outro lado, implica que incrementos da biomassa aérea sempre causam aumentos na fração coberta, como o caso do feijão (Figura 2). Forçando-se o valor unitário para $\delta$, o $\mathbf{R}^{\mathbf{2}}$ para as culturas do al godão e do milho decresceu para valores em torno de 0,7 (resultados não apresentados), com fortesubestimativa da fração coberta para baixos valores de bi omassa aérea. Como

\section{Quadro 4. Parâmetros $(\delta, \varepsilon)$ e coeficientes de determinação ajustados $\left(R^{2}\right)$ da equação [9], relacionando biomassa aérea $\left(\mathrm{Bm}_{\mathrm{d}}, \mathrm{kg} \mathrm{ha}^{-1}\right)$ e fração do solo coberta pelo dossel $\left(F_{\mathrm{d}}\right)$, para cinco culturas no Estado do Paraná}

\begin{tabular}{lccll}
\hline Cultura & $\boldsymbol{\delta}$ & $\boldsymbol{\varepsilon}$ & $\mathbf{n}^{(1)}$ & $\mathbf{R}^{\mathbf{2}}$ \\
\hline Algodão & 0,44 & $1,8 \times 10^{-2}$ & 20 & 0,94 \\
Batata & 0,86 & $9,6 \times 10^{-4}$ & 22 & 0,91 \\
Feijão & 1,01 & $7,9 \times 10^{-4}$ & 31 & 0,96 \\
Milho & 0,67 & $1,5 \times 10^{-3}$ & 38 & 0,90 \\
Soja & 0,95 & $7,0 \times 10^{-4}$ & 22 & 0,92 \\
\hline
\end{tabular}

(1) $\mathbf{n}$ é o número de pontos usados para ajuste da regressão.

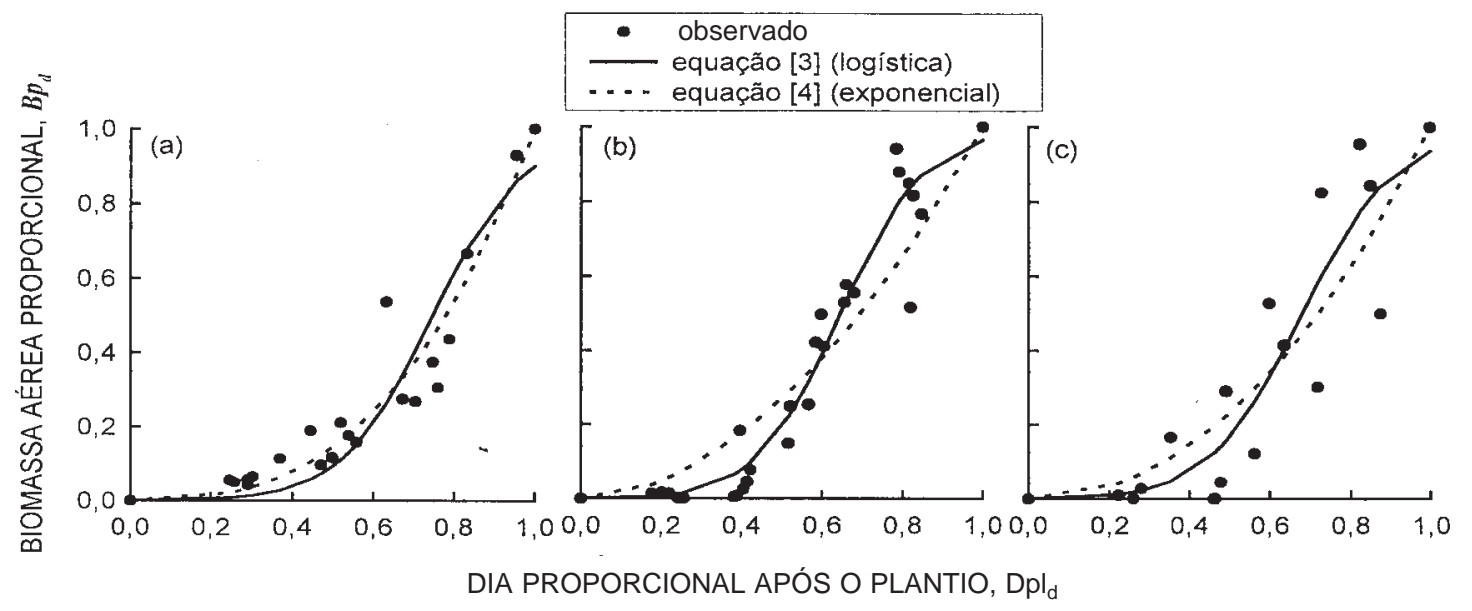

Figura 1. Relação entre biomassa aérea proporcional e dias após plantio proporcional, para as culturas do feijão (a), milho (b) e soja (c), e curvas geradas pelas equações [3] e [4]. 
a cobertura na fase inicial da cultura tem grande importância para o potencial da erosão nessa parte da safra, a inclusão da constante $\delta$ deve aumentar a precisão das estimativas nessa fase.

O desempenho das relações estabel ecidas para as culturas do feijão e do milho foi testado por meio da comparação entre a $\mathbf{F}_{\mathbf{C}_{\mathbf{d}}}$ medida e a estimada em glebas independentes. A $\mathbf{F}_{\mathbf{d}}$ estimada dependeu da variável climática utilizada (Figura 3). Para ofeijão, o uso de $\mathbf{C p l}$ apresentou desempenhomarginalmente superior, jul gando-se subjetivamente, e pel os val ores de $\mathbf{R}^{2}$ da regressão entre valores medidos e estimados (dados não apresentados). No caso do milho, não existiu diferença entre o uso de $\mathbf{C p l}$ e Dpl. Enquanto para o feijão a precisão foi similar em toda a extensão da curva, para o milho houve nítida subestimativa da fração coberta 60 dias após plantio. Esse erro é originado pelo valor do parâmetro $\delta(0,67)$, que força a ocorrência de um patamar na curva. Isso indica a necessidade de
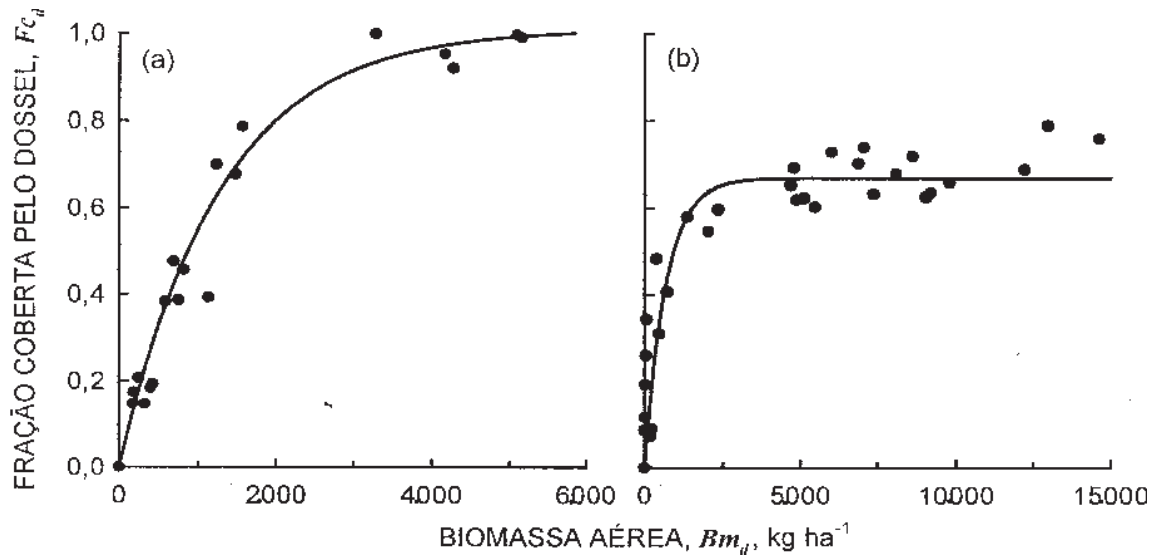

Figura 2. Relação entre a biomassa, aérea e a fração coberta pelo dossel, para as culturas do feijão (a) e do milho (b). A linha tracejada representa a equação [9] ajustada para os pontos medidos.

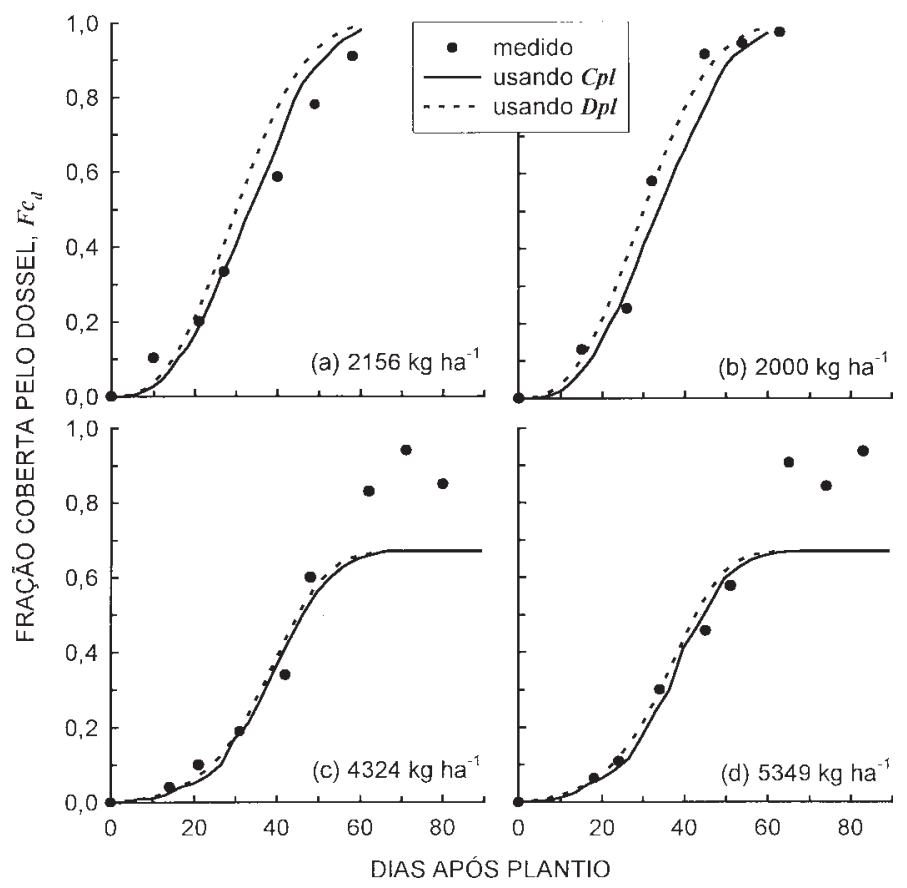

Figura 3. Comparação entre a fração do solo coberta pelo dossel medida e a calculada, usando dia proporcional após plantio ( $\mathrm{Dpl})$ ou calor acumulado proporcional após o plantio $(\mathrm{Cpl})$, para duas glebas com as culturas do feijão (a e b) e do milho (c e d), com seus respectivos rendimentos, do município de Pato Branco, Paraná. 
incluir um número maior de variedades de milho na geração do parâmetro $\delta$, uma vez que as variedades usadas no teste foram distintas daquelas usadas na geração do parâmetro(Quadro1). Esteteste émuito restrito e não permite condusões definitivas, porém indica que o método utilizado possibilita a geração de curvas de cobertura pelo dossel com um mínimo de dados locais e sua precisão é aceitável, principalmentena fase inicial das culturas, de rápido crescimento. A extrapolação das relações obtidas para outras regiões é possível porque as variáveis são proporcionais. Para isso, é necessário que sejam conhecidos os valores máximos das variáveis que caracterizam a espéciee variedade (Cx, DIx ou Dex, e $\mathbf{A x}$ ), e a população e espaçamento das culturas (Quadro 1) sejam próximos daqueles utilizados na geração dos parâmetros. Contudo, experimentos e testes mais amplos ainda são necessários para estabel ecer a confiabilidade dessas relações.

A altura mediana também apresentou boa correlação com a biomassa aérea (Quadro 5). Para a cultura da batata, ocorreu pequeno incremento nos val ores de $\mathbf{R}^{\mathbf{2}}$, quando as constantes da equação [10] foram separadas por município (resultados não apresentados). Porém, como a altura mediana é um fator secundário na determinação do efeito da cobertura sobrea erosão, não sejustifica a existência de constantes regionalizadas para apenas essa cultura, cujo porte é relativamente baixo. Para as outras culturas, não existiu diferença aparenteentre locais de amostragem.

Quadro 5. Parâmetro $(\phi)$ e coeficientes de determinação ajustados $\left(R^{2}\right)$ da equação [10], relacionando biomassa aérea $\left(\mathrm{Bm}_{\mathrm{d}}, \mathrm{kg} \mathrm{ha}^{-1}\right)$ e altura proporcional da cultura $\left(A_{d}, \mathrm{~cm}\right)$, para cinco culturas no Estado do Paraná

\begin{tabular}{lrrrr}
\hline Cultura & $\boldsymbol{\phi}$ & $\mathbf{n}^{(\mathbf{1})}$ & $\mathbf{R}^{\mathbf{2}}$ & $\mathbf{A x}^{(\mathbf{2})}$ \\
\hline & $\times 10^{-4}$ & & & $\mathrm{~cm}$ \\
Algodão & 13,0 & 20 & 0,93 & 37 \\
Batata & 9,5 & 24 & 0,87 & 27 \\
Feijão & 7,7 & 24 & 0,95 & 26 \\
Milho & 2,9 & 40 & 0,90 & 114 \\
Soja & 6,3 & 22 & 0,92 & 38 \\
\hline
\end{tabular}

(1) $\mathbf{n}$ é o número de pontos usados na regressão. ${ }^{(2)}$ Altura mediana máxima (média por cultura).

\section{CONCLUSÕES}

Os resultados demonstram ser possível estimar a fração coberta ea altura do dossel das culturas do al godão, batata, feijão, milho e soja, a partir do rendimento e de um parâmetro climático. Para tal, é necessário conhecer o índice de colheita da variedade e calor acumulado ou dias após plantio para a maturação fisiológica, de modo a gerar a relação da biomassa aérea com o tempo. Tal relação, para as culturas da soja e do milho, foi mais bem descrita por uma curva logística. Uma curva exponencial foi mais adequada para a cultura do feijão, enquanto ambas as curvas resultaram em precisão similar para as culturas do algodão e da batata. A relação entre biomassa aérea e fração coberta, de forma exponencial, deve incluir uma constante que conduza esta relação a um valor constante de fração coberta para altas quantias de biomassa, principalmente para as culturas do algodão e do milho. A altura mediana do dossel para as culturas em questão também foi adequadamente estimada por uma relação exponencial com a biomassa aérea. Testes limitados com as relações aqui apresentadas demonstraram ser possível a geração relativamente precisa da fração do solo coberta pelo dossel e da al tura das plantas, para o uso em modelagem da erosão ou planejamento conservacionista, a partir de dados históricos ou de cenários futuros.

\section{AGRADE CIMENTOS}

Aos extensionistas da EMATER (PR), M.A. de Andrade, O.R. Silva, J .E. Leitão, R.E. Dallelaste, J .B.P. de Lima, R. Kohler eA. de Souza Filho, pela coleta dos dados no campo. Aos acadêmi cos Daniele Focht e Fabiano R. de Lima, pelo auxílio na manipulação das amostras e dados.

\section{LITE RATURA CITADA}

ALBERTS, E.E.; WELTZ, M.A. \& GHIDEY, F. Plant growth component. In: LANE, L.J . \& NEARING, M.A., eds. USDA - Water erosion prediction project: hillslope profile model documentation. West Lafayette, USDA-ARS National Soil Erosion Research Laboratory. 1989. p. 8.1-8.6. (NSERL Report, 2).

DUMESNIL, D., ed. EPIC user's guide. Temple, U.S. Dept. Agr. Agr. Res. Ser. - Grassland, Soil and Water Research Laboratory, 1994 (não publicado)

ELWELL, H.A. \& STOCKING, M.A. Developing a simple yet practical method of soil loss estimation. Trop. Agric., 59:4348, 1982.

KINIRY, J .R.; WILLIAMS, J.R.; GASSMAN, P.W. \& DEBAEKE, P. A general, process-oriented model for two competing plant species. Trans. Am. Soc. Agr. Eng., 35:801-810, 1992.

RENARD, K.G.; FOSTER, G.R.; WEESIES, G.A. \& PORTER, J.P. RUSLE: Revised universal soil loss equation. J. Soil Water Conserv., 46:30-33, 1991. 
SEBER, G.A.F. \& WILD, C.J . Nonlinear regression. New York, J ohn Wiley, 1989. 768p.

SHARPLEY,A.N. \& WILLIAMS, J.R. EPIC - Erosion/Productivity Impact Calculator: 1. Model documentation. Washington, USDA, 1990. 235 p. (ARS Technical Bulletin, 1768)

STOCKING, M. Modelagem de perdas de solo: sugestões para uma aproximação brasileira. Projeto PNUD/FAO BRA/ 82011. Relatório da missão. Brasília, Ministério da Agricultura, SNPA-SRN, 1985.
SYSTAT, Inc. SYSTAT 5.02 for Windows. Evanston, 1993.

WILLIAMS, J.R.; J ONES, C.A.; KINIRY, J R. \& SPANEL, D.A The EPIC crop growth model. Trans. Am. Soc. Agr. Eng., 32:497-511, 1989.

WISCHMEIER, W.H. \& SMITH, D.D. Predicting rainfall erosion losses - a guide to conservation planning. Washington, USDA, 1978. (Agr. Hdbk., 537) 